\title{
Influence of Different Media on Micromorphology of Radiation Synthesized Poly(acrylamide-sodium acrylate) Hydrogels
}

\author{
Jun SHaN, ${ }^{\dagger}$ Zhanjun LiU, ${ }^{*}$ and Jie CHEN \\ Department of Chemistry, Tongji University, Shanghai, 200092, People's Republic of China \\ * Department of Applied Chemistry, University of Science and Technology of China, \\ Hefei, 230026, People's Republic of China
}

(Received December 22, 1997)

\begin{abstract}
Poly(acrylamide-sodium acrylate) hydrogels were synthesized by ${ }^{60} \mathrm{Co}-\gamma$ ray radiation polymerization in aqueous solutions. The micromorphology of hydrogel samples dehydrated in the different alcohol media such as methanol, ethyl alcohol, $n$-propyl alcohol, iso-propyl alcohol, and tert-butyl alcohol was observed by scanning electron microscope (SEM). The critical concentrations at volume phase transition of the hydrogels in the different alcohol medium-water solutions were obtained. The results indicate that the hydrogel samples dehydrated in methanol and in ethyl alcohol display bubble-film network structures, and those in $n$-propyl alcohol and in iso-propyl alcohol and in tert-butyl alcohol demonstrate the porous structures including the dense phase. The reason is discussed from the critical concentrations and solubility parameters of different alcohol media.

KEY WORDS Hydrogel / Micromorphology / Bubble-Film Network Structure / Porous Structure / Poly(acrylamide-sodium acrylate) / Radiation Polymerization /
\end{abstract}

A polymer hydrogel is a kind of intelligent polymer material which can modulate the swelling ratio in response to environmental stimuli such as media, ${ }^{1} \mathrm{pH},{ }^{2}$ temperature, ${ }^{3}$ and electric field. ${ }^{4}$ It has been applied to the drug release and delivery system and the chemomechanical system. ${ }^{5,6}$

In our previous work, the features of the volume phase transition of radiation synthesized poly(acrylamidesodium acrylate) hydrogels in acetone-water solutions were studied. ${ }^{7}$ The micromorphology of the bipolymer hydrogels and the polyacrylamide and poly(sodium acrylate) homopolymer hydrogels was observed by SEM. Several bipolymer hydrogels display the bubble-film network structures, and the compositions of hydrogel polymers seriously affect micromorphology. The reason was qualitatively elucidated from the two kinds of opposite forces - the hydrogen bond attraction and electrostatic repulsion which existed in the network chains of hydrogel polymers. ${ }^{8}$ The bubble-film network structures of poly(acrylamide-sodium acrylate- $\mathrm{N}$-tertbutyl acrylamide) terpolymer hydrogels were investigated. The results showed that the steric hindrance of tert-butyl groups which can weaken the hydrogen bond attraction between amide groups moderately affects the micromorphology of terpolymer hydrogels. ${ }^{9}$

In this paper, two poly(acrylamide-sodium acrylate) bipolymer hydrogels which have good mechanical strength were synthesized by the same method of ${ }^{60} \mathrm{Co}-$ $\gamma$ ray radiation polymerization. SEM was used to observe the micromorphology of the fresh cross sections of hydrogel samples dehydrated in different alcohol media. The influence of the media on the micromorphology was investigated by comparing the features of the media and the critical concentrations at volume phase transition of hydrogels.

\section{EXPERIMENTAL}

\section{Materials}

Acrylamide (AM) and sodium acrylate $(\mathrm{NaA})$ monomers and $N, N^{\prime}$-methylene bisacrylamide (MBA) crosslinking agent were used. AM was recrystallized twice in acetone solvent before use. $\mathrm{NaA}$ was prepared from acrylic acid (AA), which was purified by reduced pressure distillation. MBA was used without further purification. Several alcohol media, methanol (AR) and ethyl alcohol (AR) without purification, and $n$ - and iso-propyl alcohol (cp) and tert-butyl alcohol (cp) were distillated before use.

\section{Synthesis of $P(A M-N a A)$ Bipolymer Hydrogels by} Radiation Polymerization

The synthetic method of $\mathrm{P}(\mathrm{AM}-\mathrm{NaA})$ bipolymer hydrogels was the same as reported previously, ${ }^{8}$ and the compositions of $\mathrm{P}(\mathrm{AM}-\mathrm{NaA})$ hydrogels were characterized by FT-IR. ${ }^{10}$ The molar ratios of $\mathrm{AM} / \mathrm{NaA}$ in pregel solutions were $8.0 / 2.0$ and 7.0/3.0, respectively. The corresponding $\mathrm{P}(\mathrm{AM}-\mathrm{NaA})$ bipolymer hydrogels were called hydrogels I and II, which have good mechanical strength.

\section{Observation of Micromorphology of Hydrogels I and II by $S E M$}

SEM micrographs of hydrogels I and II were taken on a X-650 scanning electron microscope. The colorless and transparent hydrogels I and II were dehydrated for $48 \mathrm{~h}$ at room temperature in different alcohol media. Dry hydrogel samples were obtained. The gold-coated cross sections of samples were observed by SEM. SEM micrographs were shown in Figures 1 and 2.

The hydrogel I and II samples were dried slowly under infrared lamp in air for $10 \mathrm{~h}$, and became hard and transparent bars. They have perfectly similar micromorphology shown in Figure 3.

\footnotetext{
$\dagger$ To whom correspondence should be addressed.
} 


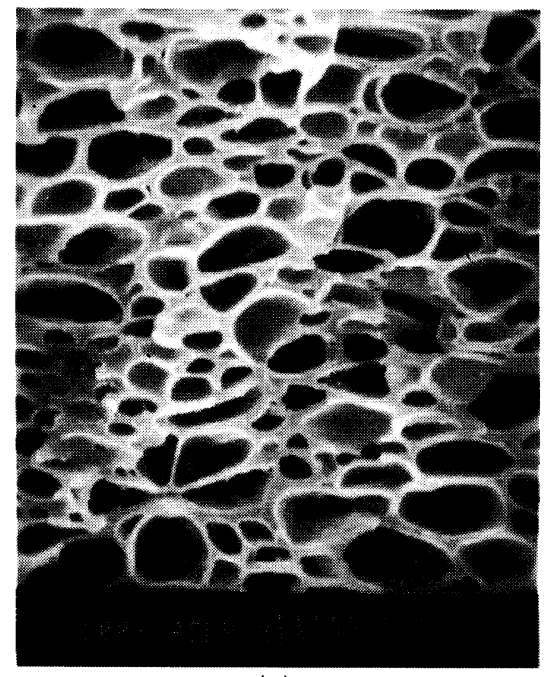

(a)

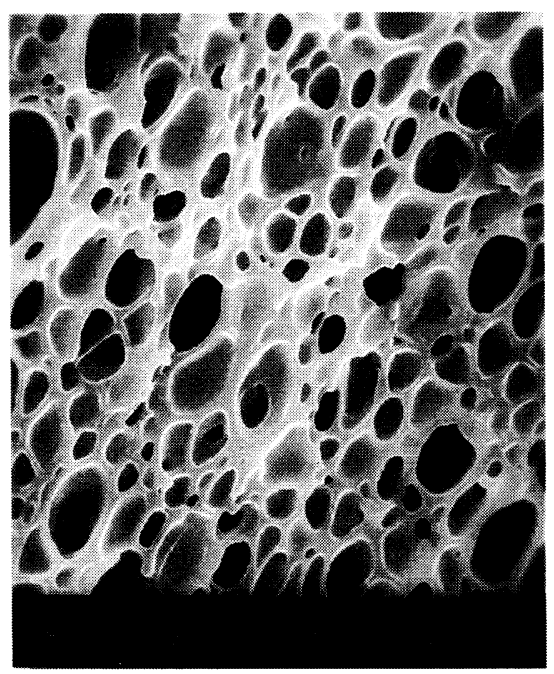

(b)

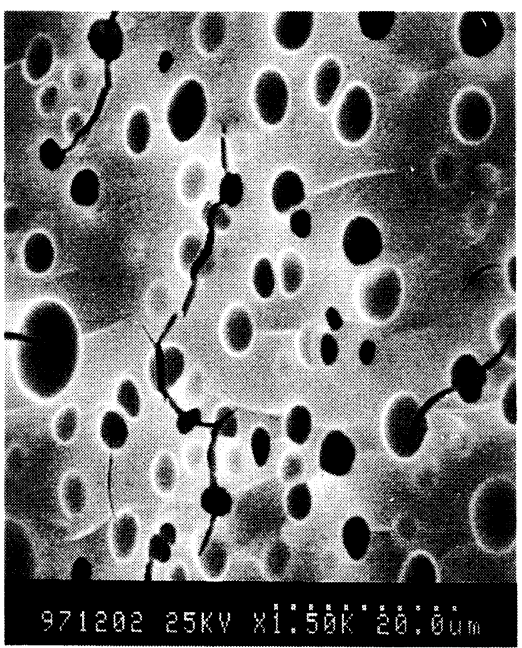

(c)

Figure 1. SEM micrographs of hydrogel I samples dehydrated respectively in methanol (a), in ethyl alcohol (b), and in $n$-and iso-propyl alcohol and tert-butyl alcohol (c).

Measurement of Curves of Swelling Kinetics of Hydrogel I in Different Alcohol Media Concentrations in Aqueous Solutions

The barlike hydrogel I was carefully cut into many

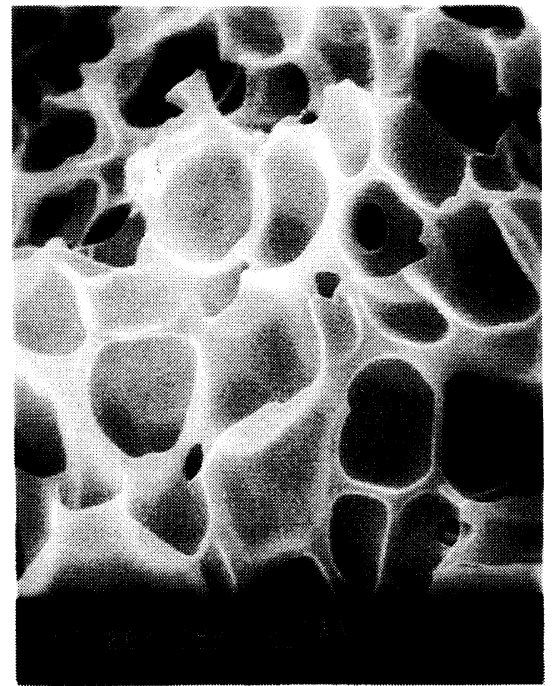

(a)

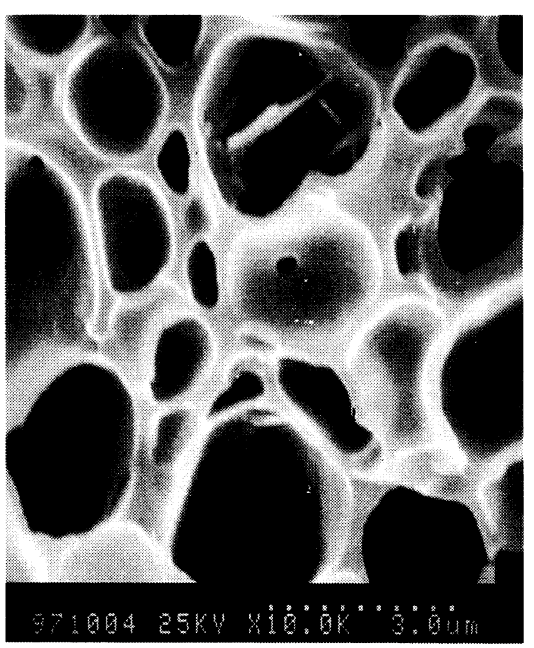

(b)

Figure 2. SEM micrographs of hydrogel II samples dehydrated in methanol (a) and in ethyl alcohol (b).

pieces about $5 \mathrm{~mm}$ long. Initial volume $\left(V_{0}\right)$ was measured by reading scope. The samples were immersed in different alcohol media concentrations in aqueous solutions at $30^{\circ} \mathrm{C}$. The swelling ratio $\phi_{\mathrm{i}}$ was determined from $\phi_{\mathrm{i}}=V_{\mathrm{i}} / V_{0}$. The curves of swelling kinetics of hydrogel I at different alcohol media concentrations in aqueous solutions were similiar to Figure 4 . A series of critical concentrations of hydrogel I samples was obtained.

\section{RESULTS AND DISCUSSION}

Influence of Different Alcohol Media on Micromorphology of $P(A M-N a A)$ Hydrogel Samples

The micromorphology of hydrogel I samples dehydrated in methanol and in ethyl alcohol displays the bubble-film network structures (see Figures 1(a) and (b)). However, the bubble-film network framwork in Figure 1(b) is thicker than that in Figure 1(a). The hydrogel I samples dehydrated respectively in $n$-propyl alcohol and in iso-propyl alcohol and in tert-butyl alcohol have very similar micromorphology as shown in Figure 1(c), i.e., porous structures. The kind of porous structure in- 
cludes the continuous and dense phase and many separate pores, which are greatly different from the above bubble-film network structure.

The hydrogel that absorbed a large amount of free water can shrink instantly as soon as it is immersed in the alcohol media, and the network chains of hydrogel bipolymer are closed up mutually and become the dense phase finally. However, as a large amount of water absorbed in hydrogel cannot be squeezed out promptly, it is very possible to cause the formation of many thoroughfares of the absorbing water, and the thoroughfares are just the pores wrapped in the dense phase in Figure 1(c).

SEM observation indicates that the micromorphology of hydrogel II in the different alcohol media is roughly

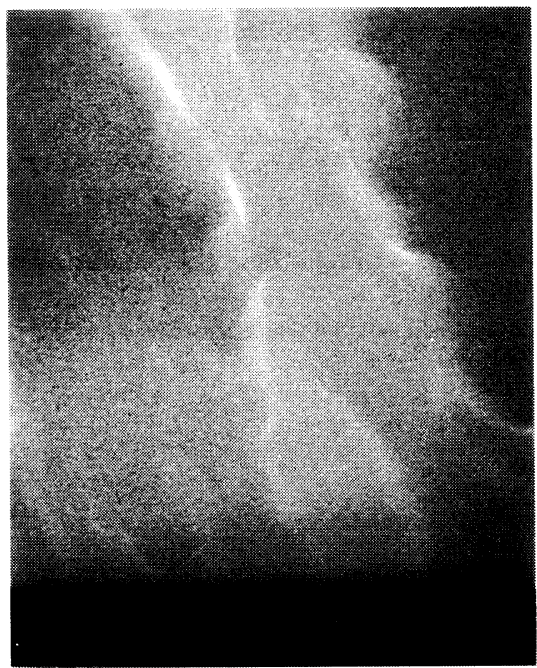

Figure 3. SEM micrograph of hydrogel I sample dried slowly in air.

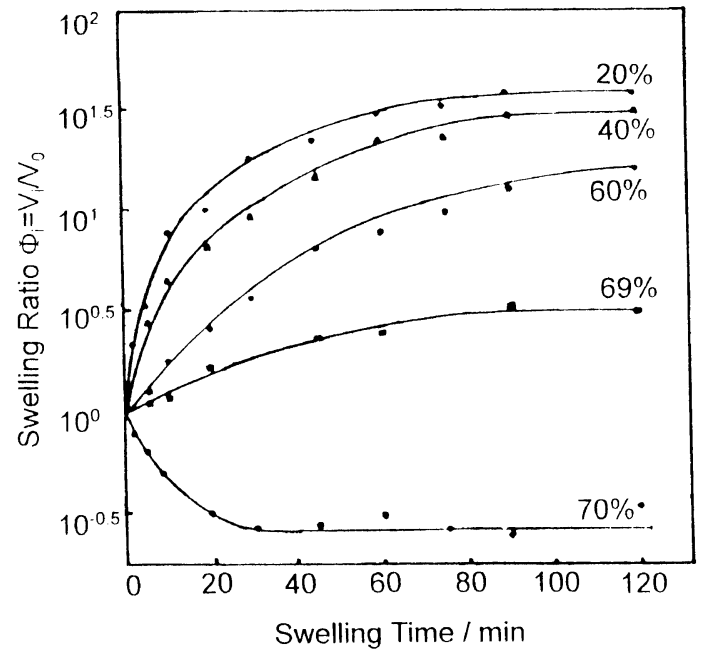

Figure 4. Curves of swelling kinetics of hydrogel I samples at different ethyl alcohol concentrations in aqueous solution: $20 \%, 40 \%, 60 \%$, $69 \%$, and $70 \%$ similar to those of hydrogel I. The hydrogel II samples dehydrated in methanol and in ethyl alcohol demonstrate the bubble-film network structures (see Figures 2(a) and (b)), and dehydrated in $n$-propyl alcohol and in iso-propyl alcohol and in tert-butyl alcohol media show the same porous structures as the hydrogel I samples shown in Figure 1(c). However, by comparison Figures 1(a) and (b) with Figures 2(a) and (b), hydrogel II in methanol and in ethyl alcohol shows a more obvious double layer bubble-film network structure than hydrogel I. According to our previous work, ${ }^{9}$ because the ratio of $\mathrm{NaA}$ unit in hydrogel II is larger than that in hydrogel I, it is attributed to the stronger electrostatic repulsion among the network chains of hydrogel II.

Obviously, though it is known alcohol media are all non-solvents for the hydrogel, their influence on the micromorphology of hydrogel samples is different because of distinct features.

\section{Micromorphology of Hydrogels I and II Samples Dried in Air}

The micromorphology of hydrogels I and II samples dried slowly in air shows much denser phase structures without any pore. It is clear that the denser phase structure is different from the porous structure. The reason why the denser phase structure is formed is that the slowly dry method of hydrogel samples is helpful to squeeze out water.

The micromorphology of the same hydrogel samples varies with the stimuli of the different factors outside hydrogel samples.

Relations between the Solubility Parameters ( $\delta$ ) of Different Alcohol Media and the Critical Concentrations $\left(C_{\mathrm{tr}}\right)$ for Hydrogel I Samples

The concentration of alcohol medium in aqueous solution where the volume phase transition of hydrogel takes place is defined as the critical concentration, $C_{\mathrm{tr}}$, which is between the maximum concentration in which hydrogel swells and the minimum concentration in which hydrogel deswells.

Table I lists the solubility of five alcohol media and water and the corresponding critical concentrations of hydrogel I.

$\mathrm{P}(\mathrm{AM}-\mathrm{NaA})$ hydrogel is a hydrophilic polyelectrolyte gel, which can be swollen but cannot be dissolved in water. The solubility parameter of the $\mathrm{P}(\mathrm{AM}-\mathrm{NaA})$ bipolymer is quite near that of water. Though the different alcohol media are easy to dissolve in water, they are non-solvents for the $\mathrm{P}(\mathrm{AM}-\mathrm{NaA})$ hydrogel. Adding the alcohol medium in water can increase the concentration of corresponding medium, and decrease the solubility $\left(\delta_{\mathrm{m}}\right)$ of the mixed solvent. The critical solubility $\left(\delta_{\mathrm{m}}{ }^{\mathrm{c}}\right)$ is defined as the solubility of mixed solvent at the corresponding critical concentration $\left(C_{\mathrm{tr}}\right)$,

Table I. Solubility $(\delta)$ and corresponding critical concentrations $\left(C_{\mathrm{tr}}\right)$ of hydrogel I samples

\begin{tabular}{|c|c|c|c|c|c|c|}
\hline Media & Water & Methanol & $\begin{array}{l}\text { Ethyl } \\
\text { alcohol }\end{array}$ & $\begin{array}{c}n \text {-Propyl } \\
\text { alcohol }\end{array}$ & $\begin{array}{c}\text { iso-Propyl } \\
\text { alcohol }\end{array}$ & $\begin{array}{l}\text { tert-Butyl } \\
\text { alcohol }\end{array}$ \\
\hline$C_{\mathrm{tr}} / \%$ & & 84.0 & 69.5 & 64.0 & 62.5 & 58.5 \\
\hline$\delta /\left(\text { cal cm }^{-3}\right)^{1 / 2}$ & 23.2 & 14.5 & 12.7 & 11.9 & 11.5 & 10.6 \\
\hline$\delta_{\mathrm{m}}^{\mathrm{c}} /\left(\mathrm{cal} \mathrm{cm}^{-3}\right)^{1 / 2}$ & & 15.9 & 15.9 & 16.0 & 15.9 & 15.8 \\
\hline
\end{tabular}


and all of $\delta_{\mathrm{m}}^{\mathrm{c}}$ of different mixed solvents are calculated by Hildebrand's equation, and are listed in Table I. It shows that they are almost the same.

The smaller the solubility of alcohol medium is, the lower is the critical concentration of corresponding alcohol medium in aqueous solution. Similarly, the more the solubility parameter of alcohol medium deviates from that of $\mathrm{P}(\mathrm{AM}-\mathrm{NaA})$ hydrogel, the more the alcohol medium affects the micromorphology of $\mathrm{P}(\mathrm{AM}-\mathrm{NaA})$ hydrogel. By combining the above investigation of SEM micrographs of hydrogels I and II with the $\delta$ of corresponding alcohol medium and the $\delta_{\mathrm{m}}{ }^{\mathrm{c}}$ of corresponding mixed solvent, the conclusions are as follows:

(1) When hydrogels I and II are in the media whose $\delta \geq 12.7\left(\mathrm{cal} \mathrm{cm}^{-3}\right)^{1 / 2}$, the micromorphology of hydrogels takes on the bubble-film network structures (or the double layer bubble-film network structures, which depend on the compositions of hydrogels).

(2) When hydrogels I and II are in the media whose $\delta \leq 11.9\left(\mathrm{cal} \mathrm{cm}^{-3}\right)^{1 / 2}$, theirs show the porous structures.

(3) It may be inferred that the micromorphology of hydrogels I and II which are in the critical phase transition where $\delta_{\mathrm{m}}{ }^{\mathrm{c}}=15.8-16.0\left(\mathrm{cal} \mathrm{cm}^{-3}\right)^{1 / 2}$ shows bubblefilm network structures.

(4) The hydrogels I and II only show the bubble-film network structures in the whole phase transition process of hydrogels in methanol or in ethyl alcohol medium aqueous solutions. However, in $n$-propyl alcohol or in iso-propyl alcohol or in tert-butyl alcohol medium aqueous solutions, the hydrogels show the bubble-film network structures $\rightarrow$ the porous structures with increase in corresponding alcohol medium concentration.

It is thus evident that the difference of the noncompatibility between the hydrogel and the medium seriously influences the micromorphology of hydrogel.

\section{REFERENCES}

1. K. Ishihara, N. Muramoto, and I. Shinohara, J. Appl. Polym. Sci., 29, 211 (1984).

2. L. C. Dong and A. S. Hoffman, J. Control. Release, 15, 141 (1991).

3. M. Yoshida, M. Asano, and M. Kumakura, Eur. Polym. J., 25, 1197 (1989).

4. S. E. Eisenberg and A. J. Grodzinsky, J. Membrane Sci., 19, 173 (1984).

5. C. M. Pai, J. Pharm. Sci., 81, 532 (1992).

6. Y. Osada and J. P. Gong, Prog. Polym. Sci., 13, 187 (1993).

7. J. Shan and Z. J. Liu, Radiat. Phys. Chem., 42, 955 (1993).

8. J. Shan, J. Chen, Z. J. Liu, and M. S. Zhang, Polym. J., 28, 886 (1996).

9. J. Shan, Z. J. Liu, F. Li, G. Zuo, J. Ji, and Y. Zhang, Polym. J., 29, 580 (1997).

10. J. Shan and Z. J. Liu, Chemical Journal of Chinese Universities, 16, 1467 (1995). 